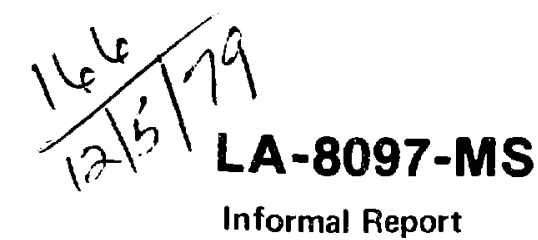

Informal Report

\section{Endochronic Viscoplasticity Model}

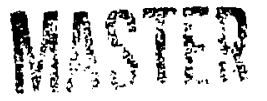

$\frac{\pi}{5}$

$\frac{1}{\infty}$

$\frac{1}{5}$

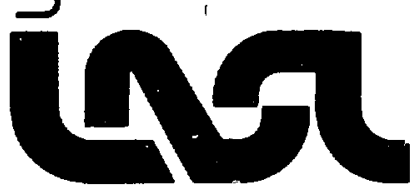


LA-8097-MS

Informal Peport

UC-71

Issued: October 1979

\title{
Endochronic Viscoplasticity Model
}

\author{
William A. Cook
}
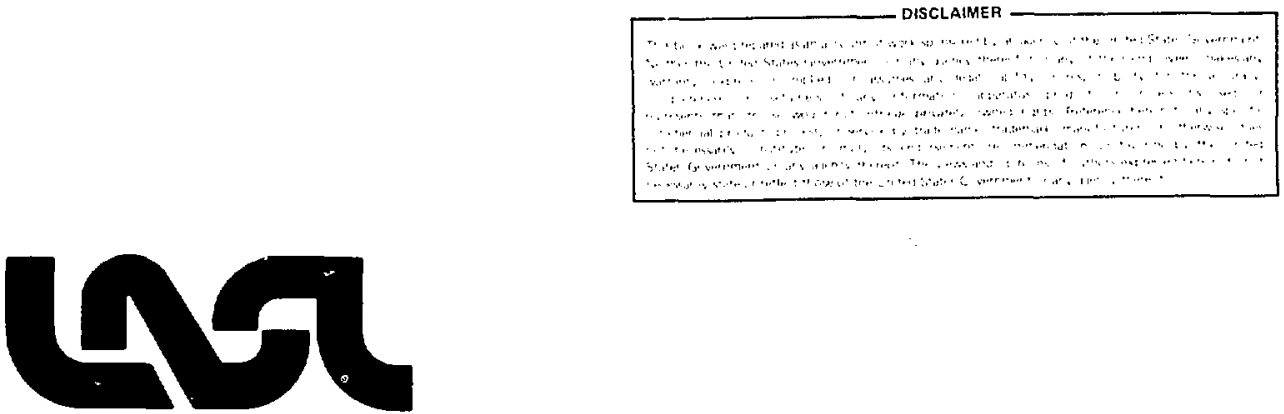


\section{MOMENCLATURE}

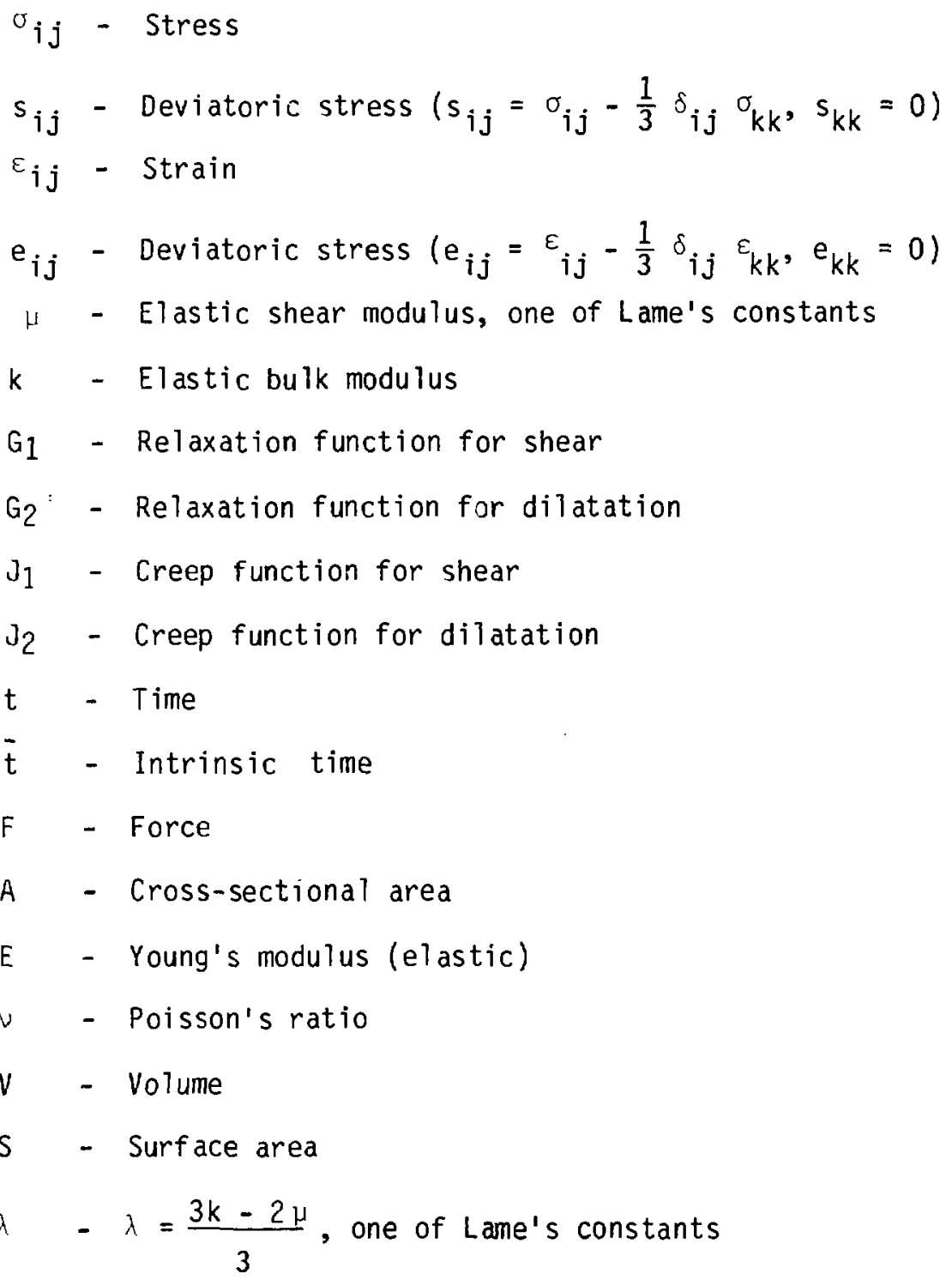

Repeated Latin indices indicate sumation $1-3$ in Secs. II and IV. 
ENDOCHRONIC VISCOPLASTICITY MODEL

by

William A. Cook

\begin{abstract}
The endochronic viscoplasticity model is presented, and the criteria for general problem analyses are discussed. Two approaches are then developed for inclusion of this model in nonlinear finite element codes. One approach includes reformulating the stiffness matrix for solution by iteration, and the other approach does not. Also, the uniaxial tension problem is studied, and the problems encountered with the use of this model are stated. Finally, recommendations are presented to check the basic postulates used to develop this model.
\end{abstract}

\title{
I. INTRODUCTION
}

The US Department of Energy, Assistant Secretary for Environment, Division of Environmental Control Technology is directing computer code development for analyzing nuclear material shipping containers that have been subjected to severe impact conditions. The endochronic viscoplasticity model was chosen as a possible model for the materials used in shipping containers. Argonne National Laboratory (ANL) was charged with the responsibility of developing this model for finite element codes. Los Alamos Scientific Laboratory (LASL) was charged with adding this model to a finite element code being developed for shipping container analysis.

The endochronic viscoplasticity model was introduced by Professor K. C. Valamis in 1971 with Ref. 1. The purpose of this model was to avoid the use of yield surfaces and thus simplify viscoplasticity analysis.

This report describes the work done at LASL with this model. In Sec. II, the model is described and discussed and in Sec. III, two approaches are 
presented for using this model in a finite element code. In Sec. IV, the uniaxial test problem is discussed along with the difficulties that are encountered in its use; and in Sec. $V$, recommendations are described for testing this model.

\section{ENDOCHRONIC MODEL}

In this section, the endochronic viscoplasticity model is developed. This development is extended from that presented in Ref. 1 to include a variable bulk modulus. We start with the basic viscoelastic constitutive relations and four basic definitions and produce an endochronic viscoplasticity model that may be used in theoretical analysis or included in a finite element analysis. This section is concluded with a discussion of the information that is required on a particular material for use in the model and on the tests that might be performed to obtain this information.

The shear viscoelastic constitutive relations ${ }^{2}$ are

$$
\begin{aligned}
& s_{i j}(x, t)=\int_{0}^{t} G_{1}(t-\tau) \frac{\partial e_{i j}(x, \tau)}{\partial \tau} d \tau, \text { and } \\
& e_{i j}(x, t)=\int_{0}^{t} J_{1}(t-\tau) \frac{\partial s_{i j}(x, \tau)}{\partial \tau} d \tau .
\end{aligned}
$$

The shear elastic relation is

$$
s_{i j}(x, t)=2 \mu e_{i j}(x, t)
$$

The dilatation viscoelastic constitutive relations ${ }^{2}$ are

$$
\begin{aligned}
& \sigma_{k k}(x, t)=\int_{0}^{t} G_{2}(t-\tau) \frac{\partial \varepsilon_{k k}(x, \tau)}{\partial \tau} d \tau \text {, and } \\
& \varepsilon_{k k}(x, t)=\int_{0}^{t} J_{2}(t-\tau) \frac{\partial \sigma_{k k}(x, \tau)}{\partial \tau} \pi \tau .
\end{aligned}
$$


The dilatation elastic relation is

$$
\sigma_{k k}(x, t)=3 k \varepsilon_{k k}(x, t)
$$

An endochronic viscoplasticity model can be defined as follows.

(1) Replace the time $(t)$ in the viscoelastic constitutive relation with a new variable, intrinsic time $(\bar{t})$.

(2) Define the intrinsic time $(\bar{t})$ as

$$
d \bar{t}=\frac{d \zeta}{1+\beta \zeta}
$$

where $\beta$ is a constant and

where $(\beta d \zeta)^{2}=K_{1} d \varepsilon_{i j} d \varepsilon_{j j}+K_{2} d \varepsilon_{i j} d \varepsilon_{i j}$ and $d \zeta \geq 0$.

$K_{1}$ and $k_{2}$ are either constants or functions of strain invariants. If $k_{1}$ and $k_{2}$ are functions of strains and not strain invariants, a change in coordinate definition would alter the calculated behavior of the material.

(3) Assume a relaxation function for shear of

$$
G_{1}(\bar{t})=2 \mu e^{-\alpha \bar{t}},
$$

where $\alpha$ is a constant.

(4) Assume a creep function for dilatation of

$$
J_{2}(\bar{t})=\frac{1}{3 k} e^{-\gamma \bar{t}}
$$

where $Y$ is a constant. 
From endochronic viscoplasticity definitions (1) and (3),

$$
s_{i j}(x, \bar{t})=\int_{0}^{\bar{t}} 2 j e^{-\alpha(\bar{t}-\tau)} \frac{\partial e_{i j}(x, \tau)}{\partial \tau} d \tau .
$$

Differentiating this equation with respect to $\bar{t}$ (see Ref. 3, page 312,313 ),

$$
\frac{\partial s_{i j}(x, \bar{t})}{\partial \bar{t}}=-a s_{i j}(x, \bar{t})+\frac{2 \mu \partial e_{i j}(x, \bar{t})}{\partial \bar{t}} .
$$

This equation can be written in incremental form as

$$
\Delta s_{i j}=-\alpha s_{j j} \Delta \bar{t}+2 \mu \Delta e_{i j} .
$$

From endochronic viscoplasticity definition (2), the intrinsic time can be written incrementally as

$$
\Delta \overline{\mathrm{t}}=\frac{\Delta \zeta}{1+\beta \zeta}
$$

Thus Eq. (12) can be written as

$$
\Delta s_{i j}=-\alpha s_{i j} \frac{\Delta \zeta}{1+\beta \zeta}+2 \mu \Delta e_{i j} .
$$

When the definitions for deviatoric stress and strain are substituted into Eq. (14),

$$
\begin{aligned}
\Delta \sigma_{i j} & =-\left(\frac{\alpha \Delta \zeta}{1+\beta \zeta}\right)\left(\sigma_{i j}-\frac{1}{3} \delta_{i j} \sigma_{k k}\right)+2 \mu\left(\Delta \varepsilon_{i j}-\frac{1}{3} \delta_{i j} \Delta \varepsilon_{k k}\right) \\
& +\frac{1}{3} \delta_{i j} \Delta \sigma_{k k} .
\end{aligned}
$$


From endochronic viscoplasticity definition (1) and (4),

$$
\varepsilon_{k k}(x, \bar{t})=\int_{\delta}^{\bar{f}} \frac{e^{-\gamma(\bar{t}-\tau)}}{3 k} \frac{\partial \sigma_{k k}(x, \tau)}{\partial \tau} d \tau .
$$

Differentiating this equation with respect to $\overline{\mathrm{t}}$ (see Ref. 3, p. 312,313 ),

$$
\frac{\partial \varepsilon_{k k}(x, \bar{t})}{\partial \bar{t}}=-i \varepsilon_{k k}(x, \bar{t})+\frac{1}{3 k} \frac{\partial \sigma_{k k}(x, \bar{t})}{\partial \bar{t}} .
$$

This equation may be written in incremental form as

$$
\Delta \varepsilon_{k k}=-Y \varepsilon_{k k} \Delta \bar{t}+\frac{1}{3 k} \Delta \sigma_{k k}
$$

Equation (13) can be used to eliminate the intrinsic time in Eq. (18) and give

$$
\Delta \sigma_{k k}=3 k \Delta \varepsilon_{k k}+3 k\left(\frac{\gamma \Delta \zeta}{1+\beta \zeta}\right) \varepsilon_{k k}
$$

Using Eq. (19), $\Delta \sigma_{k k}$ may be eliminated in Eq. (15) to give the endochronic viscoplasticity relation

$$
\begin{aligned}
\Delta \sigma_{i j} & =-\frac{\alpha \Delta \zeta}{1+\beta \zeta}\left(\sigma_{i j}-\frac{1}{3} \delta_{i j} \sigma_{k k}\right)+2 \mu \Delta \varepsilon_{i j}+\delta_{i j}\left(\frac{3 k-2 \mu}{3}\right) \Delta \varepsilon_{k k} \\
& +\delta_{i j} k\left(\frac{\gamma \Delta \zeta}{1+B \zeta}\right) \varepsilon_{k k} .
\end{aligned}
$$

For $\alpha$ and $\gamma$ defined as

$$
\alpha=3\left[\alpha_{1}+\delta_{i j}\left(\alpha_{2}-\alpha_{1}\right)\right] \beta \text { and } \gamma=3 \gamma_{1} \beta \text {, }
$$


Eq. (20) can be written as

$$
\begin{aligned}
\Delta \sigma_{i j} & =-3\left[\alpha_{1}+\delta_{i j}\left(\alpha_{2}-\alpha_{1}\right)\right] \frac{\beta \Delta \zeta}{1+\beta \zeta}\left(\sigma_{i j}-\frac{1}{3} \delta_{i j} \sigma_{k k}\right)+2 \mu \Delta \varepsilon_{i j} \\
& +\delta_{i j}\left(\frac{3 k-2 \mu}{3}\right) \varepsilon_{k k}+\delta_{i j} 3 k \gamma_{1}\left(\frac{\beta \Delta \zeta}{1+\beta \zeta}\right) \varepsilon_{k k} .
\end{aligned}
$$

The term $\frac{\beta \Delta \zeta}{1+\beta \zeta}$ is obtained from the incrementa) equation (see endochronic viscoplastic definition (2) or Eq. (7))

$$
(\beta \Delta \zeta)^{2}=K_{1}\left(\Delta \varepsilon_{k k}\right)^{2}+K_{2} \Delta \varepsilon_{i j} \Delta \varepsilon_{i j}
$$

Equation (19) can be written in terms of $\gamma_{1}$ as

$$
\Delta \sigma_{k k}=3 k \Delta \varepsilon_{k k}+9 k \gamma_{1}\left(\frac{\beta \Delta \zeta}{1+\beta \tau}\right) \varepsilon_{k k}
$$

To solve any particular problem, we need to know the following material properties for Eqs. (22), (23), and (24): elastic properties $k$ and $\mu$, and endochronic viscoplasticity properties $\alpha_{1}, \alpha_{2}, \gamma_{1}, k_{1}$, and $k_{2}$.

For this endochronic viscoplasticity model to be a general viscoplasticity model, these endochronic viscoplasticity properties must be independent of any problem, dependent only on material. An inspection of Eqs. (22), (23), and (24) suggests the following:

(1) a hydrostatic compression test,

(2) a tensile test,

(3) a shear test, and

(4) a triaxial test.

The hydrostatic test would determine $\gamma_{1}$, the tensile test would determine $a_{1}$, the shear test would determine $\alpha_{2}$, the tensile and shear tests would determine $K_{1}$ and $K_{2}$, and the triaxial test would be a check to determine whether the endochronic viscoplasticity model is a general model. 
In this section I develop two possible approaches for using the endochronic viscoplasticity model in a fincite element code. Both approaches use iteration for the solution of the nonlinearity caused by this nonlinear material model. Model 1 does not reform the master stiffness, whereas Model 2 reforms the stiffness but probably converges more rapidly in the iteration scheme.

The endochronic model, Eq. (22) of Sec. II, can be written in matrix notation as

$$
\{\Delta \sigma\}=[D]\{\Delta \varepsilon\}-f(\zeta)[G]\{\sigma\}+f(\zeta)[H]\{E\},
$$

where

$$
\begin{aligned}
& f(\zeta)=\frac{\beta \Delta \zeta}{1+\beta \zeta}, \\
& \{\dot{\Delta \sigma}\}^{\top}=\left(\begin{array}{llllll}
\Delta \sigma_{11} & \Delta \sigma_{22} & \Delta \sigma_{33} & \Delta \sigma_{12} & \Delta \sigma_{23} & \Delta \sigma_{31}
\end{array}\right), \\
& \{\Delta \varepsilon\}^{\top}=\left({ }_{11} \varepsilon_{12} \Delta \varepsilon_{22} \Delta \varepsilon_{33} \Delta \varepsilon_{12} \Delta \varepsilon_{23} \Delta \varepsilon_{31}\right), \\
& \{\sigma\}^{\top}=\left(\begin{array}{llllll}
\sigma_{11} & \sigma_{22} & \sigma_{33} & \sigma_{12} & \sigma_{23} & \sigma_{31}
\end{array}\right), \\
& \{E\}^{\top}=\left(\begin{array}{llllll}
\varepsilon_{11} & \varepsilon_{22} & E_{33} & \varepsilon_{12} & E_{23} & \varepsilon_{31}
\end{array}\right), \\
& {[\mathrm{D}]=\left[\begin{array}{cccccc}
\lambda+2 \mu & \lambda & \lambda & 0 & 0 & 0 \\
\lambda & \lambda+2 \mu & \lambda & 0 & 0 & 0 \\
\lambda & \lambda & \lambda+2 \mu & 0 & 0 & 0 \\
0 & 0 & 0 & 2 \mu & 0 & 0 \\
0 & 0 & 0 & 0 & 2 \mu & 0 \\
0 & 0 & 0 & 0 & 0 & 2 \mu
\end{array}\right],}
\end{aligned}
$$




$[G]=a_{1}\left[\begin{array}{rrrrrr}2 & -1 & -1 & 0 & 0 & 0 \\ -1 & 2 & -1 & 0 & 0 & 0 \\ -1 & -1 & 2 & 0 & 0 & 0 \\ 0 & 0 & 0 & 1 & 0 & 0 \\ 0 & 0 & 0 & 0 & 1 & 0 \\ 0 & 0 & 0 & 0 & 0 & 1\end{array}\right]$, and

$[H]=3 k \gamma,\left[\begin{array}{cccccc}1 & 1 & 1 & 0 & 0 & 0 \\ 1 & 1 & 1 & 0 & 0 & 0 \\ 1 & 1 & 1 & 0 & 0 & 0 \\ 0 & 0 & 0 & 0 & 0 & 0 \\ 0 & 0 & 0 & 0 & 0 & 0 \\ 0 & 0 & 0 & 0 & 0 & 0\end{array}\right]$.

Let the index $j$ indicate the $j$ th time, and let $k$ indicate the finite element increment (repeated indices do not indicate sumation in this section). Then the virtual work relation for finite element analysis may be written as

$$
\begin{aligned}
& \sum_{k=1}^{K}\left(\int_{V_{k}}\{\Delta \sigma\}_{j k}^{T} \delta\{\Delta \varepsilon\}_{j k} d V-\int_{V_{k}}\{\Delta F\}_{j k}^{T} \delta\{\Delta u\}{ }_{j k} d V\right. \\
& \left.-\int_{S_{k}}{ }^{[\Delta T\}^{T}{ }_{j k} \delta\{\Delta u\}} d S\right)=0,
\end{aligned}
$$

where $\{\Delta F\}$ are the body and or inertia loads, $\{\Delta T\}$ are the incremental traction loads, and $\{\Delta \mathrm{u}\}$ are the incremental displacements. 
Equation (1) can be written as

$$
[\Delta \sigma\}_{j}=[D]\{\Delta \varepsilon\}_{j}-f\left(\zeta_{j}\right)[G]\{\sigma\}_{j}+f\left(\zeta_{j}\right)[H]\{\varepsilon\}_{j} .
$$

Two methods were investigated for including the endochronic model in the NONSAP code. 4

Method 1: Substitute Eq. (27) into Eq. (26)

$$
\begin{aligned}
& \sum_{k=1}^{K}\left(\{\Delta \bar{u}\}_{j k}^{\top} \int_{V_{k}}[B]^{\top}[D][B] d y-\int_{V_{k}}\{\sigma\}_{j k}^{\top}[G] f\left(\zeta_{j k}\right)[B] d V\right. \\
& +\int_{V_{k}}\{\varepsilon\}_{j k}^{\top}[H] f\left(\zeta_{j k}\right)[B] d V-\int_{V_{k}}\{\Delta F\}_{j k}^{\top}[N] d V \\
& \left.-\int_{S_{k}}[\Delta T\}_{j k}^{T}[N] d V\right) \quad \delta\{\Delta \bar{u}\}_{j k}=0 \text {, }
\end{aligned}
$$

where $[N]$ is the shape function. Thus

$$
\begin{aligned}
& \{\}_{j k}=[B]\{\Delta \bar{u}\}_{j k}, \quad \text { and } \\
& \{\Delta u\}_{j k}=[N]\{\Delta \bar{u}\}_{j k} .
\end{aligned}
$$

The first term in Eq. (28) is the stiffness matrix; note that it is independent of $j$, whereas the next four terms that compose the force vector are dependent on $j$. However, the last two terms are independent of stress $\{\sigma\} j$, strain $\{\varepsilon\}_{j}$, or intrinsic time $\zeta_{j}$. Thus Eq. (28) can be written as

$$
\left\{F_{1 j}\left([E\}_{j},[\sigma\}_{j}, \zeta_{j}\right)+\Delta F_{2 j}\right\}=[K][\Delta U\}_{j},
$$

where $\{\Delta U\}$ is the matrix of all nodal values, $\left\{\Delta F_{1 j}\left(\{\varepsilon\}_{j},\{\sigma\}_{j}, \zeta_{j}\right)+\right.$ $\left.\Delta^{\mathrm{C}}{ }_{2 j}\right\}$ is the matrix of all incremental forces, and $[K]$ is the master stiffness 
matrix. Equation (29) can be solved by iteration. Iteration is the same as incrementing, only the incremental loads are zero. The main virtue of this method is that the stiffness needs to be formulated and reduced only once.

Method 2: The stress and strain can be written as

$$
\begin{aligned}
& \{\sigma\}_{j}=\{\sigma\}_{j-1}+\{\Delta \sigma\}_{j} \text {, and } \\
& \{\varepsilon\}_{j}=\{\varepsilon\}_{j-1}+\{\Delta \varepsilon\}_{j},
\end{aligned}
$$

where $\{\sigma\}_{j-1}$ and $\{\varepsilon\}_{j-1}$ are known from the last iteration. Substituting these equations into Eq. (27),

$$
\{\Delta \sigma\}_{j}=[\bar{D}]_{j}\{\Delta \varepsilon\}_{j}-[\bar{G}]_{j}\{\sigma\}_{j-1}+[\bar{H}]_{j}\{\varepsilon\}_{j-1}
$$

where

$$
\begin{aligned}
& {[\bar{D}]_{j}=\left([I]+f\left(\zeta_{j}\right)[G]\right)^{-1}\left([D]+f\left(\zeta_{j}\right)[H]\right),} \\
& {[\bar{G}]_{j}=\left([I]+f\left(\zeta_{j}\right)[G]\right)^{-1} f\left(\zeta_{j}\right)[G],} \\
& {[\bar{H}]_{j}=\left([I]+f\left(\zeta_{j}\right)[G]\right)^{-1} f\left(\zeta_{j}\right)[H] \text {, and }} \\
& {[I] \text { is } 6 \times 6 \text { identity matrix. }}
\end{aligned}
$$

Substituting Eq. (31) into the virtual work Eq. (26),

$$
\begin{gathered}
\sum_{k=1}^{K}\left(\{\Delta \bar{u}\}_{j k}^{\top} \int_{V_{k}}[B]^{\top}[\bar{D}]_{j}[B] d V-\int_{V_{k}}\{\sigma\}_{j-1, k}^{\top}[\bar{G}]_{j}[B] d V\right. \\
+\int_{V_{k}}\{\varepsilon\}_{j-1, k}^{T}[\bar{H}]_{j}[B] d V-\int_{V_{k}}[\Delta F\}_{j k}^{\top}[N] d V
\end{gathered}
$$




$$
\left.-\int_{S_{k}}\{\Delta T\}_{j k}^{T}[N] d S\right) \delta\{\Delta \bar{u}\}_{j k}=0 .
$$

This equation can be written as

$$
\left\{F_{1 j}\left(\zeta_{j}\right)+F_{2 j} j=\left[K\left(\zeta_{j}\right)\right]\{\Delta U\}\right.
$$

Not.ce with this method that both the force vector and stiffness matrix must be reformulated with each increment or iteration. Again, Eq. (33) can be solved for each increment by iteration. The virtue of this method is that it would probably be more stable. A method similar to this was used in Ref. 5 for creep analysis.

Because of its simplicity, Model 1 was coded into the NONSAP code for axisymmetric continuum elements. To check this Model, I analyzed a cylinder in tension. The stress-strain results from this analysis were quite different from those expected, so I decided to study the behavior of the endochronic viscoplasticity model with the uniaxial problem.

\section{UNIAXIAL PROBLEM}

For the uniaxial problem shown in Fig. 1, the following as sumptions apply.
(1) Normal stress
$\sigma_{11}=F / A, \sigma_{22}=0$. , and $\sigma_{33}=0$.
(2) Shearing stresses
$\sigma_{12}=0 ., \quad \sigma_{23}=0 .$, and $\sigma_{31}=0$.
(3) Shearing strains
$\varepsilon_{12}=0 ., \quad \varepsilon_{23}=0$. , and $\varepsilon_{31}=0$.

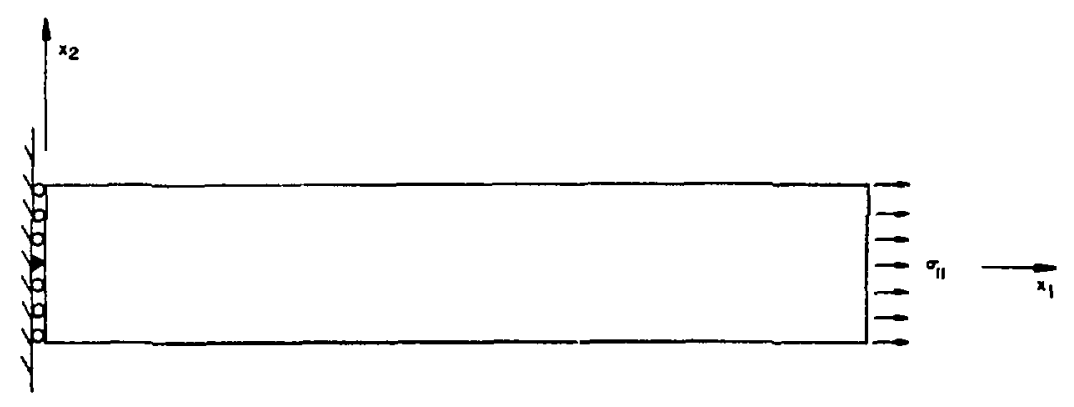

Fig. 1.

Uniaxial problem. 
From these assumptions for the uniaxial problem and Eqs. (22), (23), and (24) in $\mathrm{Sec}$. II, the endochronic viscoplasticity re? ation can be derived as

$$
r_{11}=E \therefore_{11}-2(1+\nu) \alpha_{1}\left(\frac{B \Lambda \zeta}{1+B_{i}}\right) \sigma_{11}+E_{1}\left(\frac{B \Delta \zeta}{1+B \zeta}\right) r_{k k} \text {, }
$$

where

$$
\begin{aligned}
& 22+\therefore_{33}=-2\left\{v i c 11+\frac{(1-2 v)(1+\nu)}{E} \alpha_{7}\left(\frac{\beta \Delta \zeta}{1+\beta \zeta}\right) \sigma_{11}\right. \\
& \left.+(1+v) r_{1}\left(\frac{B \Delta \zeta_{5}}{1+\beta \tau}\right) k k\right\}, \\
& (z \ldots)^{2}=k_{1}\left(\Delta \varepsilon_{k k}\right)^{2}+k_{2}\left(\Delta \varepsilon_{11}^{2}+\Delta \varepsilon_{22}^{2}+\Delta \varepsilon_{33}^{2}\right) \text {, and } \\
& \therefore \sigma_{11}=3 k \wedge \varepsilon_{k k}+9 k \gamma_{1}\left(\frac{\beta \Delta \zeta}{1+\beta \zeta}\right) \varepsilon_{k k} \text {. }
\end{aligned}
$$

A computer code has been written to solve Eqs. (34) and (35) and is included in the report as the Appendix.

Consider the aluminum alloy

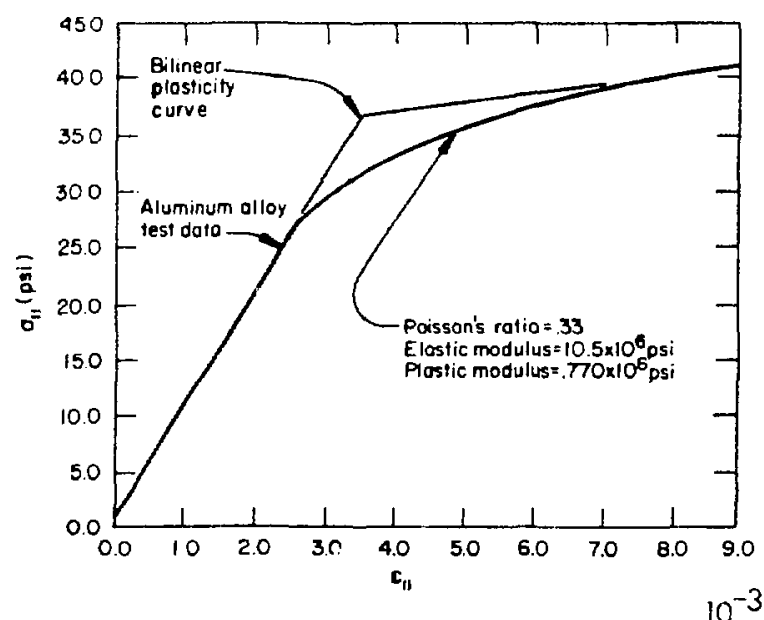
material discussed in Ref. 6, page 173. A stress strain relation for this material is shown in Fig. 2 .

Reference 7 , page 16 , states "that the yielding of a metal is, to a first approximation unaffected by a hydrostatic pressure or tension." Thus, let $\gamma_{1}$ be zero. Also, assume $K_{2}$ equal to zero, and then Eq. (35) can be written as

Fig. 2.

$$
\beta \Delta \zeta=\frac{\sqrt{k_{1}}}{3 k} \Delta \sigma_{11} \text {. }
$$

Stress $\left(\sigma_{11}\right)$ strain $\left(\varepsilon_{11}\right)$ relation for aluminum alloy uniaxial problem. 
This equation can be integrated to give

$$
\beta \zeta=\frac{\sqrt{\mathrm{K}_{1}}}{3 \mathrm{k}} \sigma_{11} .
$$

Substituting Eqs. (37) and (38) into Eq. (34) gives

$$
\Delta \sigma_{11}=E \Delta \varepsilon_{11}-2(1+\nu) \alpha_{1} \frac{\left(\sqrt{\mathrm{K}_{1}} / 3 \mathrm{~K}\right) \Delta \sigma_{11} \sigma_{11}}{\left.1+\left(\sqrt{\mathrm{K}_{1}} / 3 \mathrm{~K}\right) \sigma_{11}\right)} .
$$

For $\sigma_{11}=40500$ psi in Fig. 2,

plastic modilus $=\frac{\Delta \sigma_{11}}{\Delta \varepsilon_{11}}$.

A 1so, for $\sigma_{11}=40500 \mathrm{psi}$, and $K_{1}=15555$,

$1+\frac{\sqrt{k_{1}}}{3 k} \sigma_{11}: \frac{\sqrt{k_{1}}}{3 k} \sigma_{11}$ and Eq. 39 reduces such that Eq. 40 becomes

$$
\text { plastic modulus }=\frac{E}{1+2(1+\nu) \alpha_{1}} \text {. }
$$

From the values in Fig. 2,

$$
\alpha_{1}=4.74 \text {. }
$$

Using the following data for the code in the Appendix, I generated Fig. 3 .

$$
\begin{aligned}
& \text { NINC }=30 \\
& \text { NIT }=5 \\
& \text { EMOD }=10.5 \times 10^{6} \text { psi } \\
& \text { BETA1 }=15555 \\
& \text { ALPHA1 }=4.74 \\
& \text { DELP }=4190 \text { lbs }
\end{aligned}
$$




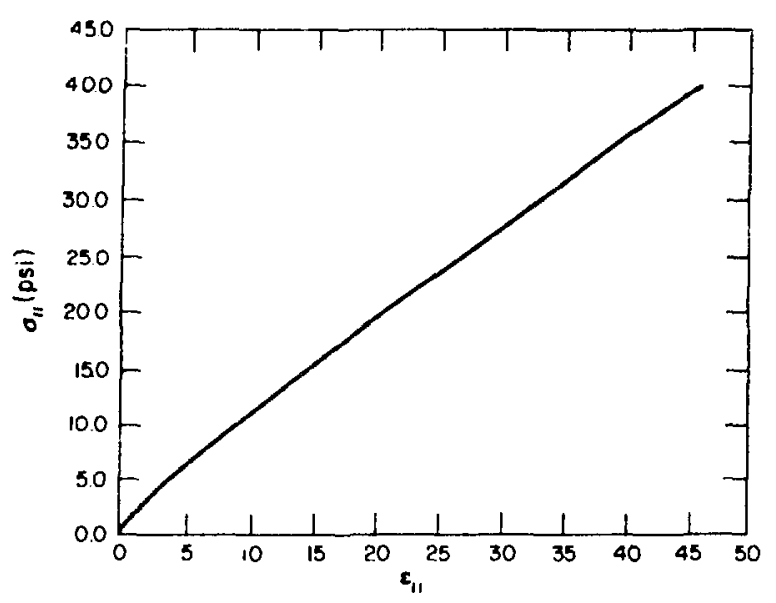

Fig. 3.

Endochronic model for aluminum alloy.

$$
\begin{aligned}
& \text { EL. }=10 \mathrm{in.} \\
& \text { AREA }=3.0 \mathrm{in}^{2} \\
& \text { ECVGE }=.00001 \\
& \text { ENU }=.333 \\
& \text { GAM1 }=0.0 \\
& \text { BETA2 }=0.0
\end{aligned}
$$

Figures 3 and $i$ do not agree. An examination of the calculation revealed that $\Delta k_{k k}$ was constant and did not allow the model to react as expected. Note that if one example $c$ an be found that can not be sulved with the endochronic model presented, then the model is not a general viscoplasticity model. The fact that Fig. 3 does not model the aluminum alloy in Fig. 2 does not imply that it is impossible. There are many other possibilities. Thus many values of $\alpha_{1}$ and $\beta_{1}$ were used with little improvement in the results. Another approach used was to set $\beta_{1}=0$ and use many values for $\alpha_{1}$ and $\beta_{2}$. These results were better; however, $\beta_{2}$ is needed for shear data, so this approach is not entirely satisfactory. Yet another approach that might be tried is to let $k_{1}$ and $k_{2}$ be functions of strain invariants in the code and see if better results can be calculated.

Another concern is that for the uniaxial problem, assumptions (1) and (2) are stress boundary conditions while assumption (3) is a limiting condition on the displacements. If assumption (3) were not used, would a more physical solution be forthcoming? Would such a solution overcome the difficulties seen with the present solution?

This uniaxial problem has not shown that the endochronic viscoplasticity is at a general model. However, the difficulty encountered in trying to use it $=z$ jjoh a simple problem makes the model's generality questionable. If

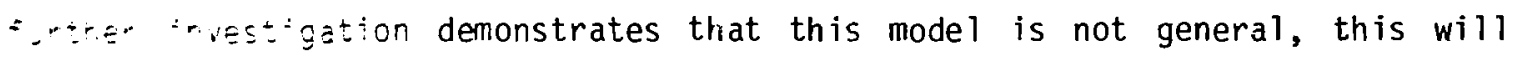
$\because \because \because \cdots, z:$ rtrinsic time is not a material property. 
V. RECOMMENDATIONS

My recommendations follow.

(1) Do the testing discussed in Sec. II. I believe that the triaxial test would show that instrinsic time is not a material property and that the endochronic viscoplasticity model is not a general viscoplasticity model. If this is the case, perhaps intrinsic time could be changed until it is a material property. Dr. B. J. Hsieh of ANL shows some very impressive results when he modifies the intrinsic time definition in Refs. 8 and 9. I think much more work is needed in this area of correlation of the endochronic model with testing.

(2) Develop theoretical endochronic codes for tests specimen, like the code presented in the Appendix. Also, develop a nonlinear least-squares code to be used to fit the test data with the theoretical model.

\section{REFERENCES}

1. K. C. Valanis, "A Theory of Viscoplasticity Without a Yield Surface, Part I, General Theory, Part II, Application to Mechanical Behavior of Metals," Archives of Mechanics 23, $517-551$ (1971).

2. R. M. Christensen, "Theory of Viscoelasticity: An Introduction," (Academic Press, New York, 1971).

3. C. R. Wylie, Advanced Engineering Mathematics 4th Ed. (McGraw-Hill, New York, 1975).

4. K. J. Bathe, E. L. Wilson, and R. H. Iding, "NONSAP - A Structural Analysis Program for Static and Dynamic Response of Linear Systems," Dept. of Civil Eng., Univ. of California report USCESM 74-3 (February 1974).

5. C. A. Anderson, "Cavity Stability: Finite Element Analysis for Steady and Transient Creep," Los A Iamos Scientific Laboratory report LA-5769 (February 1975).

6. F. R. Shanley, Strength of Materials (McGraw-Hill, New York, 1957).

7. R. Hill, The Mathematical Theory of Plasticity (Oxford at the Clarendon Press, 1950).

8. B. J. Hsieh, "On the Uniqueness and Stability of Endochronic Plasticity Theory," Argonne National Laboratory report ANL-CT-78-51 (September 1978). 
9. B. J. Hsieh, "The Use of Endochronic Plasticity For Multi-Dimensional Small and Large Strain Problems," Argonne National Laboratory report ANL-CT-79-19 (March 1979).

\section{APPENDIX}

VARIABLES INPUT

NINC - Number of load increments

NIT - Maximum number of iterations allowed for convergence of endochronic viscoplasticity relation

EMOD - E (Young's Modulus)

BETA1 - $B_{1}=\left(K_{1}\right)^{1 / 2}$

ALPHA $1-\alpha_{1}$

DELP - $\triangle p$ (load increment)

EL $\quad-\quad \ell$ (length of bar in Fig. 1)

AREA - A (cross sectional area of bar in Fig. 1)

ECVGE - Criterion for convergence of endochronic viscoplastiticy relation

ENU - $\nu$ (Poisson's ratio)

GAM1 $-\gamma_{1}$

BETA2 $-\beta_{2}^{1}=\left(K_{2}\right)^{1 / 2}$ 
LASL Identification No.LP-2024.

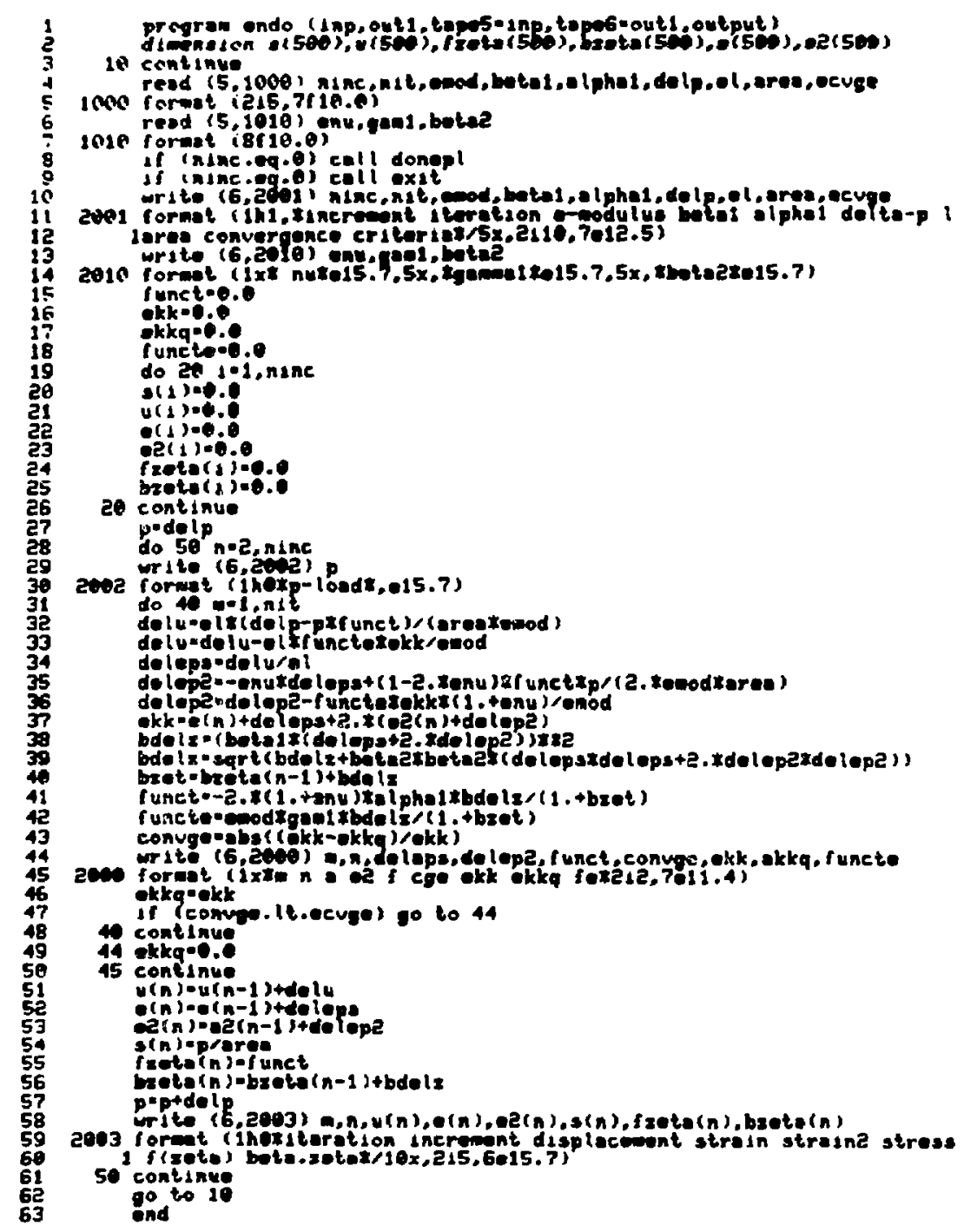

